Documentation et bibliothèques

DOCUMENTATION BIBLIOTHEQUES

\title{
Vers une philosophie de la bibliothéconomie
}

\section{Gilles Caron}

Volume 21, numéro 4, décembre 1975

URI : https://id.erudit.org/iderudit/1055381ar

DOI : https://doi.org/10.7202/1055381ar

Aller au sommaire du numéro

\section{Éditeur(s)}

Association pour l'avancement des sciences et des techniques de la documentation (ASTED)

\section{ISSN}

0315-2340 (imprimé)

2291-8949 (numérique)

Découvrir la revue

Citer cet article

Caron, G. (1975). Vers une philosophie de la bibliothéconomie. Documentation et bibliothèques, 21(4), 187-196. https://doi.org/10.7202/1055381ar

\section{Résumé de l'article}

Après avoir tracé l'historique de la profession de bibliothécaire, l'auteur propose une nouvelle identification du bibliothécaire axée sur l'objet même de la bibliothéconomie, c'est-à-dire l'information enregistrée, et préconise une révision des structures et des comportements individuels dans le sens d'un professionnalisme mieux perçu des individus qui y oeuvrent.
Tous droits réservés $@$ Association pour l'avancement des sciences et des techniques de la documentation (ASTED), 1975
Ce document est protégé par la loi sur le droit d'auteur. L’utilisation des services d'Érudit (y compris la reproduction) est assujettie à sa politique d'utilisation que vous pouvez consulter en ligne.

https://apropos.erudit.org/fr/usagers/politique-dutilisation/ 


\section{Vers une philosophie de la bibliothéconomie}

\section{Gilles Caron}

Conseiller au développement des ressources documentaires (sciences sociales) Université Laval, Québec

Après avoir tracé l'historique de la profession de bibliothécaire, l'auteur propose une nouvelle identification du bibliothécaire axée sur l'objet même de la bibliothéconomie, c'est-à-dire l'information enregistrée, et préconise une révision des structures et des comportements individuels dans le sens d'un professionnalisme mieux perçu des individus qui y œuvrent.

The author outlines the history of the profession of librarian and proposes a new identity for the librarian based on the very subject of library science, that is recorded information. He advocates that the structures and behaviour of the individuals be revised to induce more discerning professionalism on the part of those concerned.

Después de haber dado un cuento histórico de la profesión de bibliotecario, el autor propone una nueva identificación de bibliotecario que tiene más relación con el sujeto de la ciencia bibliotecaria, o sea información inscrita, y recomienda una revisión de estructuras y comportamientos individuales que resultarán en un profesionalismo mejor visto por los que en esto trabajan.

\section{Image et réalité}

L'éternel débat sur le professionnalisme qui secoue la bibliothéconomie depuis le début des années trente est, en soi, une illustration de ce conflit entre l'image et la réalité, entre ce qu'on est et ce qu'on voudrait être, de ce mal-être collectif qui affecte les bibliothécaires depuis presque deux générations.

Pendant que certains groupes accédaient, ne serait-ce que juridiquement, presque instantanément au statut tant recherché, ${ }^{1}$ le bibliothécaire, héritier d'une profession hautement séculaire, piétinait et piétine encore, accroché à un statut que certains sociologues désignent du terme savant de "semi-profession ${ }^{2}{ }^{2}$

1. C'est le cas, par exemple, des chiropraticiens, denturologues et physiothérapeutes.

2. Voir à ce sujet William J. Goode, "The theoretical limits of professionalization». in Amitai Etzioni, ed., The Semi-professions and their Organization; Teachers, Nurses, Social Workers, New York, Free Press, 1969, p. 285 .
Plusieurs raisons expliquent cet état de choses. Cependant, l'une des plus importantes paraît être la permanence d'une contradiction (qui afflige la profession) entre l'image que l'on désire projeter et la réalité, c'est-àdire le comportement de fait du bibliothécaire $œ u v r a n t$ dans l'univers qui est le sien. ${ }^{3}$

Alors que, dans la plupart des professions perçues comme telles aujourd'hui, le statut professionnel est apparu comme l'affirmation publique d'une situation de fait acquise antérieurement suite à un bagage de connaissances spécialisées, une situation de service perçue comme hautement désirable par la population, ou simplement suite à des attitudes propres et conformes à celles de l'élite professionnelle, dans le cas du bibliothécaire la situation est tout autre: la recherche du statut y apparaît souvent comme une fin en soi, un moyen d'atteindre à on ne sait trop quoi sans avoir à se redéfinir dans ce que l'on

3. Leo N. Flanagan, "Professionalism dismissed?" College and Research Libraries, vol. 34, no. 3 (May 1973), 209. 
est et ce que l'on fait, ou un moyen de perpétuer encore plus longtemps le statu quo existant et de s'accorder une certaine sécurité psychologique et matérielle sans laquelle le changement deviendrait inévitable. Ainsi, cette recherche du statut professionnel apparaît souvent comme le dénouement ultime d'une vaste opération de camouflage qui vise à consacrer la situation de l'image au détriment de la réalité.

La réalité est loin d'être aussi professionnelle qu'on voudrait bien le croire. Comme individu, qu'on le veuille ou non, le bibliothécaire est encore perçu, par la population en général, comme "la personne qui travaille dans une bibliothèque». D'ailleurs, ceci inclut souvent toutes les personnes, depuis le directeur jusqu'au balayeur. Ceci est en soi l'illustration d'un échec, de cette incapacité pour le bibliothécaire de se définir en terme de service perçu et accepté comme tel par la population plutôt qu'identifié au lieu physique où s'exerce généralement le service en question. Personne n'osera pourtant identifier le médecin à la "personne qui travaille dans un hôpital».

Cette situation, d'ailleurs, n'est pas exclusive au bibliothécaire. Le pharmacien, par exemple, représente le cas type d'un autre professionnel qui éprouve des problèmes d'images, tiraillé qu'il est entre l'image "service professionnel» qu'il veut se donner et l'image "maison d'affaires" que lui prêtent beaucoup de gens. ${ }^{4} \mathrm{Ce}$ problème entraîne d'ailleurs, ici-même au Québec, des conflits certains au sein de la profession. La différence entre le pharmacien et le bibliothécaire réside dans le fait que le premier reconnaît le problème tandis que nous, nous nous sentirions ridicules de seulement soulever la question.

La réalité de la profession prise dans son ensemble n'est pas des plus exaltantes. Les salaires versés y sont relativement faibles, compte tenu des exigences académiques requises pour y accéder. La capacité de nonréaction de la dite profession face aux stress externes, ce que Georges Cartier appelle gentiment «le niveau de tolérance élevé du bibliothécaire ${ }^{5}$ pourrait à elle seule faire l'objet

4. Norman K. Danzin and Curtis J. Mettlin, «Incomplete professionalization: the case of pharmacy", Social Forces, vol. 46, no. 3 (March 1968), 375-381.

5. Georges Cartier, «Pour un engagement socio-politique des bibliothécaires québécois", Documentation et bibliothèques, vol. 19, no 3 (septembre 1973), 103. d'une tragi-comédie d'un goût douteux. Les cas récents, ici au Québec, de la politique du livre et de la situation des bibliothécaires de cégeps sont autant de cas types de non-réaction professionnelle des plus remarquables. À part quelques lettres publiées à l'occasion dans Le Devoir et de nombreux commentaires entendus dans le milieu fermé des bibliothèques, ce fut le calme plat. II n'y eut rien à la radio, ni à la télévision, aucune conférence de presse, pas même la plus petite démonstration, ne serait-ce que symbolique, devant le monument aux morts ou la statue de la reine Victoria. Rien. Sans vouloir être méchant, c'est vraiment la seule profession où de tels événements se produisent et entraînent si peu de réactions publiques.

La réalité du bibliothécaire œuvrant au sein de la bibliothèque ne constitue certainement pas non plus l'illustration la plus représentative du comportement professionnel type. La réalité de la bibliothèque, en particulier de ce qu'on appelle la "grosse bibliothèque", qu'on le veuille ou non, est celle d'une structure hautement hiérarchisée et bureaucratisée, souvent repliée sur elle-même et ses petites habitudes où trop souvent les idées et les décisions sont le monopole d'une élite administrative restreinte. ${ }^{6}$

De fait, le type de structure existant au sein de la grande bibliothèque traditionnelle va souvent à l'encontre de tout comportement professionnel des individus y travaillant. ${ }^{7}$ L'identification aux buts et aux objectifs de la profession qui devrait guider chacun des individus qui s'affirment professionnels est le plus souvent complètement annihilée au profit des buts poursuivis par l'organisation perçue comme une fin en soi; ainsi, chacun se perçoit, non comme un professionnel offrant un service, mais comme un élément, un rouage servant les fins de l'organisation.

Plusieurs auteurs traitent longuement des conflits susceptibles de surgir, au sein d'organisations dites non-professionnelles, entre les professionnels avec les normes et objectifs de leur profession et l'organisation avec ses nor-

6. À souligner que ceci n'a rien de péjoratif. Paul Wasserman dans The New Librarianship: A Challenge for Change, New York, Bowker, 1972, 8-11, fait l'analyse appliquée à la bibliothèque américaine de ce processus naturel de vieillissement qui reste le propre de toute organisation d'importance.

7. Mary Lee Bundy and Paul Wasserman, «Professionalism reconsidered". College and Research $\mathrm{Li}$ braries, vol. 29, no. 1 (January 1968), 16. 
mes et objectifs propres. ${ }^{8} \mathrm{C}$ 'est, par exemple, le problème du contentieux d'un organisme quelconque, des ingénieurs œuvrant au gouvernement ou des médecins pratiquant dans les hôpitaux. Tous partagent au sein de chacune de leur profession respective certaines normes de qualité professionnelle applicables au service offert, normes qui, souvent, entreront en conflit avec les objectifs poursuivis par l'organisation dans laquelle ils œuvrent.

Dans le cas de la bibliothèque, ce problème ne se pose pas. De fait, c'est l'organisation qui suggère le rôle joué et assumé par chacun des acteurs. Le bibliothécaire de référence fait de la référence, le catalogueur catalogue, le spécialiste de la sélection sélectionne. Toutes ces personnes entrant dans une organisation annihilent leur qualité de professionnel au profit d'un rôle organisationnel. Bien plus, au lieu de retrouver ces divers "professionnels" réunis autour de certaines normes et objectifs professionnels tel que ça se passe généralement chez les membres d'une même profession, on les retrouve le plus souvent en conflit plus ou moins larvé les uns avec les autres (catalogue versus référence, etc.), pour le plus grand bien de l'organisation qui, de par la structure qu'elle maintient, profite nécessairement de cette attitude non-professionnelle des individus.

\section{Evolution historique récente}

Cette réalité de non-professionnel qu'assume quotidiennement le bibliothécaire est fondée sur un certain nombre de contradictions ou, plus exactement, de confusions longtemps entretenues sur ce qu'est, en définitive, l'objet du bibliothécaire. Sur cette confusion s'est érigée une longue histoire de maladresses et d'erreurs qui, d'ailleurs, persistent encore aujourd'hui. Mais, quel est-il cet objet?

Pour Mason, ${ }^{9}$ le bibliothécaire s'identifie à un certain nombre d'opérations qui ont pour point central la "science de l'information». Voici le schéma qu'il a développé:

8. Voir Ronald M. Pavalko, Sociology of Occupations and Professions, Itasca, III., Peacock, 1971, p. 188192; George Ritzer, Man and his Work: Conflict and Change, New York, Appleton-Century-Crofts, 1972, p. 85-96; Richard W. Scott, "Professional employees in a bureaucratic structure: social work» , in Amitai Etzioni, ed., The Semi-professions and their Organization; Teachers, Nurses, Social Workers, New York, Free Press, 1969, p. 82-140.

9. Donald Mason, "Librarianship and information work, one discipline?", Library Association Record, vol. 72, no. 9 (September 1970), 310.



En résumé, pour Mason, il se greffe autour du concept d' "Information science" un certain nombre d'activités qui recoupent celles généralement dévolues aux bibliothécaires, documentalistes, «information scientists", etc.

Pour Turick, "The library is an information center; we are the information profession". ${ }^{10}$ Cette dernière citation appelle peu de nuances, avouons-le. Cependant, la réalité, encore là, ne présente pas de la profession une image tout aussi consistante d'elle-même. Beaucoup de bibliothécaires, à cause de la structure dans laquelle ils évoluent ou simplement à cause de leur tempérament propre, se présentent encore aujourd'hui comme engagés dans un processus qui a pour point central d'intérêt la bibliothèque et non pas l'information.

Ceux que certains identifient à des «information workers" 11 ou "information specialists " ${ }^{12}$ apparaissent souvent beaucoup plus préoccupés de satisfaire aux besoins propres de l'organisation qu'ils représentent que de jouer pleinement leur rôle "d'agent de com-

10. Dorothy Ann Turick, "If we wait", Reference Quarterly, vol. 12, no. 4 (Summer 1973), 342. Les mots soulignés ne sont pas de nous.

11. Paul Wasserman, The New Librarianship..., p. 220.

12. Herbert Schur, "What educational programme is required to produce the necessary specialists?", in 2 . Education of Information Specialists for the 1970's, Aslib Proceedings, vol. 24, no. 10 (October 1972), 574. Schur souligne, entre autres, que le terme «information specialists" inclut de larges secteurs de la bibliothéconomie moderne. 
munication dans un réseau de moyens de communications modernes. ${ }^{13}$

Le bibliothécaire doit faire un choix entre la bibliothèque et ce qu'elle représente et l'information. Ce dilemne n'est pas, comme on pourrait être porté à le penser à première vue, artificiel et dénué d'intérêt. C'est un problème réel, un problème qui remonte aux origines mêmes de la profession, et la cause de nombreuses frustrations au sein de la profession. ${ }^{14}$

Historiquement, comme le souligne JeanRémi Brault, il y eut depuis toujours identification entre l'imprimé, l'information, la bibliothèque et, bien sûr, le bibliothécaire:

"La bibliothèque, depuis toujours, s'est identifiée à une sorte de monopole de l'information [...] Cette information était tout entière contenue dans l'imprimé. Celui-ci, tout naturellement, se réfugiait dans la bibliothèque. ${ }^{15}$

Le problème s'est déjà posé lorsque l'information a cessé d'être le monopole exclusif de l'imprimé. Dès ce moment, s'est établi un premier clivage entre les partisans de la bibliothèque, centre de l'information, et ceux de la bibliothèque, refuge de l'imprimé. Cette étape, quoi qu'on en dise, la profession ne l'a pas encore assumée pleinement. Pendant que les deux écoles de bibliothéconomie du Québec, par exemple, présentent des programmes de cours qui tentent d'inclure un éventail aussi large que possible des diverses sources documentaires, et ce, indépendamment du support, certains auteurs et non des moindres nous présentent des définitions de la profession qui laissent songeur. Ainsi Shera pense que:

«En tant qu'activité professionnelle, la bibliothéconomie, au sens général du terme, englobe l'ensemble des organismes, opérations, techniques et principes qui contribuent à rendre les documents graphiques aussi utiles à la société que

13. Reynald Rivard, "La communication humaine et l'évolution du rôle du bibliothécaire", in Association canadienne des bibliothécaires de langue française, 25 e congrès annuel. Ste-Adèle-en-Haut, 14-15 octobre 1969, Information, communication et bibliothèque, Montréal, ACBLF, 1970, p. 37

14. Voir à ce sujet Paul Wasserman, The New Librarianship..., p. 197; Jesse H. Shera, The Foundations of Education for Librarianship, New York, Wiley, 1972, p. 171

15. Jean-Rémi Brault, "La documentation et ses langages", Documentation et bibliothèques, vol. 20 , no 3 (septembre 1974), 123. cela est humainement possible, c'est-àdire à maximiser l'utilité sociale que présentent les documents graphiques. ${ }^{16}$

II faut lire Shera pour enfin percevoir que, selon lui, "graphic records" peut aussi vouloir dire, à la limite, document audio-visuel, un élément sur lequel malheureusement il ne s'attarde pas beaucoup. ${ }^{17}$

Heureusement, tous les auteurs ne présentent pas de définitions de l'objet de la bibliothéconomie qui soient aussi restrictives sinon dans le fond, au moins dans la lettre. Ainsi, pour Shores, l'objet de la bibliothéconomie devient le «knowledge most significant to mankind" ${ }^{18}$, et pour Mukherjee, les "recorded communications" $\cdot{ }^{19}$ On peut être plus ou moins d'accord avec la formulation qu'ils en présentent, mais au moins leur objet $n$ 'est-il aucunement restrictif et, en ce sens, ils restent fidèles à ce qu'a pu être depuis toujours l'objet de la profession, et ce, indépendamment du support.

Cette difficulté du bibliothécaire à assumer l'information sur un support autre qu'imprimé $n$ 'est pas que théorique. Pratiquement, force nous est de reconnaître que l'intégration du non-imprimé n'est souvent que très artificiellement assurée au sein de la bibliothèque. La floraison de vidéothèques, audiovidéothèques et cinémathèques au sein comme à l'extérieur des bibliothèques permet difficilement de percevoir la bibliothèque comme chapeautant le tout.

C'est à ce moment que prend fin cette identification nécessaire qui existait auparavant entre le bibliothécaire, la bibliothéconomie et la bibliothèque. Le bibliothécaire $œ u v r a n t$ dans une vidéothèque (un élément du champ de la connaissance ou de l'information) ne peut plus et ne veut plus être identifié à des méthodes appropriées à un support donné, l'imprimé à un lieu privilégié, la bibliothèque.

Le problème, aujourd'hui, c'est le terme même de "bibliothécaire», naturellement

16. Jesse $H$. Shera, "Bibliothéconomie, documentation et science de l'information", Bulletin de l'Unesco à l'intention des bibliothèques, vol. 22, no 2 (mars 1968), 67.

17. Jesse $H$. Shera, Sociological Foundations of Librarianship, New York, Asia Pub. House, 1970, p. 30.

18. Louis Shores, "A Philosophy of librarianship", Library and Information Science, no. 9 (1971), 41

19. Ajit Kumar Mukherjee, Librarianship, its philosophy and history, New York, Asia Pub. House, 1966, 4. 
identifié à la bibliothèque comme si le lieu physique et ce qu'il implique comme approche et technique permettait encore de cerner avec précision l'objet même de la profession.

Certains ont tenté d'abolir cet anachronisme entre deux termes qui ne coincident plus, soit en rejetant l'information non-graphique du champ de la bibliothéconomie, soit encore en niant à l'information non-graphique toutes particularités. Ainsi, on soumettait l'information non-graphique aux normes et aux techniques propres à la bibliothèque (document graphique), d'où des tensions qui souvent ont poussé les personnes œuvrant dans ces secteurs à rejeter à la fois la bibliothèque et leur qualité de bibliothécaires. ${ }^{20}$

En un mot, si l'on accepte que l'information, et plus particulièrement l'information enregistrée, ${ }^{21}$ soit l'objet du bibliothécaire, on doit accepter que le bibliothécaire puisse œuvrer sur des supports variés, avec des méthodes diverses, et ce, au sein de bibliothèques ou ailleurs. Pour résumer, il faut détacher le bibliothécaire et la bibliothéconomie d'un lieu physique qui ne représente plus l'ensemble des activités professionnelles de ce dernier.

Si le bibliothécaire, donc, tient à revenir aux sources, à ce qu'il était effectivement avant l'arrivée du non-imprimé, il aurait avantage à s'identifier non plus au contenant, la bibliothèque, mais au contenu, l'information en-

20. Jacques Demers, "Les bibliothèques se meurent: vive les centres de documentation!», Bulletin de l'ACBLF, vol. 18, no 1 (mars 1972), 36-50. Jacques Demers, par exemple, s'identifie comme audiovidéothécaire

21. Discuter de supports, c'est discuter d'informations enregistrées. Pour nous, cela va de soi, l'information dite enregistrée constitue l'objet minimum du bibliothécaire. Mais peut-on et doit-on aller plus loin? Le bibliothécaire de référence qui indique de mémoire à un utilisateur le nom de la personne-ressource la plus apte à répondre à ses besoins, le bibliothécaire qui consulte par téléphone un confrère ou le spécialiste qui détient l'information joue-t-il un rôle de bibliothécaire? Personnellement et avec d'autres, nous croyons que oui, et souvent beaucoup plus efficacement qu'en ne comptant que sur l'information pré-enregistrée. Alors, où se termine le rôle du bibliothécaire? Nous avons déterminé la limite inférieure de son champ d'activité, l'information enregistrée. La limite supérieure paraît, elle, encore à définir. Le bibliothécaire, c'est un spécialiste de l'information enregistrée, et plus. Voir Maurice Bernard Line, "The social scientist and his information needs". in Patricia Layzell Ward, ed. Proceedings of the 16th Annual Conference and Study Group, London. The Library Association, 1968, p. 15; Mary Lee Bundy and Paul Wasserman, "Professionalism reconsidered"..., 9. registrée. ${ }^{22}$ Autrement, il risque de perpétuer la confusion concernant le service qu'il offre effectivement et se place ainsi dans une situation strictement défensive où, petit à petit, avec l'apparition continuelle de nouveaux supports, son rôle dans le champ de l'information ne pourra aller qu'en s'amenuisant.

Cette erreur historique qui a vu nos prédécesseurs s'identifier, eux et leur profession, à un lieu physique fut plus tard accentuée par un ensemble de techniques développées pour les besoins du type d'informations alors disponibles et du lieu physique qui devait les contenir, la bibliothèque. En un temps où la bibliothèque se percevait d'abord et avant tout comme dépositaire de la connaissance, celleci étant nécessairement traduite sur l'imprimé, où l'information ainsi emmagasinée y trouvait un caractère immuable et se cumulait lentement et où les directeurs d'aujourd'hui s'appelaient "conservateurs", la bibliothèque $a$ développé des normes et techniques s'adaptant aux documents traités et aux exigences de l'époque. Tout le monde a en mémoire la période glorieuse de la classification de Dewey (1876), suivie de la classification décimale universelle (C.D.U.) (1905) et de celle de la Library of Congress (L.C.) (1899-1920), une époque, il faut le dire, extrêmement fructueuse intellectuellement pour la profession, une époque où la connaissance pouvait encore être présentée comme un tout universel, une période où le savoir encyclopédique était encore relativement possible. Cette époque n'est pourtant pas si loin de nous, à peine 80 ans!

Et pourtant depuis lors, tout a été bouleversé tant en terme de types ou supports d'informations disponibles qu'en qualité et en quantité. Nous n'insisterons pas sur les vieux clichés qui veulent que maintenant le volume d'informations disponibles ait atteint des proportions dantesques, ${ }^{23}$ que la connaissance

22. Nous sommes donc personnellement en désaccord avec toute définition qui tend à identifier le bibliothécaire à la bibliothèque. Ainsi, pour Asheim: "The term "librarian" (is used) to designate those who are qualified by background and training to go beyond the level of analysis of established rules and techniques to the analysis of library problems and the formulation of original and creative solutions for them. "Voir Lester Eugene Asheim, "Education and manpower for librarianship", ALA Bulletin, vol. 62, no. 9 (October 1968), 1096.

23. Toffler, par exemple, souligne qu'aujourd'hui le nombre de périodiques et articles scientifiques double tous les quinze ans et que ce rythme s'accélère sans cesse. Voir Alvin Toffler, Le choc du futur, Paris, Denoël, 1971, p. 43. 
qui en est le produit plus ou moins direct se cumule parallèlement en des périodes de temps de plus en plus courtes, etc.

Quoi qu'il en soit, cette connaissance qui se cumule aussi rapidement est le fruit d'un flux énorme d'informations, informations caractérisées par un temps de demi-vie sans cesse plus court. L'information, qu'on le veuille ou non, est maintenant une chose passagère, éphémère par essence. La population le perçoit comme tel, bombardée constamment qu'elle est par ce que l'on appelle "la nouvelle", nouvelle que demain l'on oubliera pour la remplacer par une plus récente. "La mémoire est une faculté qui oublie", et aujourd'hui plus que jamais sous peine d'intoxication à brève échéance.

Dans le monde académique, la situation est sensiblement la même, sinon pire. Dès que l'information est signalée, on se l'arrache, chacun s'efforçant de la traduire en connaissance, et on publie rapidement quelque chose de peur d'être devancé par quelqu'un d'autre.

Comment le bibliothécaire, ce spécialiste de l'information enregistrée, a-t-il fait face à ce nouveau défi? Très mal, il faut l'avouer. Le bibliothécaire a continué clopin-clopant à appliquer les normes et techniques issues d'une autre époque, comme auparavant. Encore aujourd'hui, dans la plupart des bibliothèques académiques, par exemple, on considère comme fort respectable un délai de trois mois avant de rendre disponible un ouvrage après son arrivée à la bibliothèque. II était donc évident que, pour le chercheur confronté à un milieu éminemment compétitif, de tels délais apparaissaient et apparaissent encore aujourd'hui comme inadmissibles, et avec raison.

Le bibliothécaire, qui avait raté son entrée dans le monde du non-imprimé, ratait aussi son entrée dans le monde de l'information éphémère. Le besoin étant là, d'autres individus, cela va de soi, devaient se charger de combler les lacunes auxquelles le bibliothécaire ne pouvait, ou plutôt, avait négligé de faire face; d'où l'apparition de nouveaux personnages, un peu plus ouverts, qui élaborèrent d'autres approches perçues comme plus aptes à relever le défi en y adjoignant les produits les plus récents que cette civilisation de l'éphémère avait créés.

Ces nouveaux individus, documentalistes, «information scientists», analystes de l'information, eurent tôt fait de se tailler une place enviable, de s'installer en maîtres dans un champ que les bibliothécaires, n'eût été leur incapacité de réaction assez phénoménale, auraient dû de toute logique occuper. ${ }^{24}$ Parce qu'il n'avait su faire face au changement, accroché qu'il était à un ensemble de normes et techniques issues d'une autre époque, le bibliothécaire se voyait encore une fois amputé d'une partie de son rôle comme spécialiste de l'information enregistrée, et ce, au profit d'un nouveau groupe d'individus un peu plus au fait des besoins réels de l'utilisateur.

II n'existait cependant pas d'oppositions réelles entre les deux groupes. ${ }^{25}$ Les deux se percevaient comme œuvrant sur un même produit, l'information enregistrée, avec peutêtre des approches différentes. Qui plus est, quoique en retard, le bibliothécaire se sentit obligé d'absorber certaines des approches utilisées ou développées par les documentalistes et autres. Par exemple, les systèmes de dissémination de l'information, qu'ils soient "off-line» ou "on-line" sont des produits de l'approche par descripteurs maintenant acceptée par le bibliothécaire.

Mais sans contredit, ce qui impressionna le plus le bibliothécaire dans toute cette révolution fut la venue de l'ordinateur. Conscient d'avoir pris du recul, le bibliothécaire vit dans cet instrument le moyen de régler tous ses maux, de rattraper le temps perdu dans son rôle de pourvoyeur de l'information.

Malheureusement, et contrairement aux documentalistes et aux autres spécialistes qui, eux, avaient tenté de créer de nouvelles ap-

24. Shera considère, à ce propos, "an unfortunate use of terminology" la monopolisation par les «information scientists" de l' «information theory": "Mais l'expression sonne bien et ceux qui s'occupaient de faciliter l'accès au savoir enregistré s'en emparèrent très vite. C'est ainsi qu'on en arriva bientôt à employer l'expression "science de l'information" pour désigner la bibliothéconomie de type non traditionnel.» Voir: Jesse H. Shera, «Bibliothéconomie..., " 67

25. Pour Mason, les deux groupes participent, de fait, à une seule et même profession: "The fact that there is little to choose between the services offered leads me to believe that there is only one profession, though I would hesitate to give it a name. Comparisons with other professions are not necessarily valid, but I think we can just glance at the medical profession, where a doctor can be engaged in any of a whole range of activities, from research to general practice, and yet still be a member of one profession. I would suggest that the field of information activities presents a similar picture and that it should be possible to be a member of one professional body yet be engaged in basic research into information science, or applied research into systems, or in specialized or general information and library services. Voir: Donald Mason, "Librarianship...", 310 . 
proches en intégrant à ceux-ci les moyens techniques les plus à point, le bibliothécaire se contenta et se contente encore trop souvent de traduire en langage machine des techniques et approches héritées du $19 \mathrm{e}$ siècle. Aucune discussion sur les principes de base, aucune réflexion sur les "pourquoi?». Plutôt que de repenser les méthodes héritées qui ne correspondent plus nécessairement aux besoins de l'époque, on a trop souvent tendance à solutionner le problème en mécanisant systématiquement les dites opérations, comme si la machine, à elle seule, pouvait suppléer aux déficiences organiques des systèmes appliqués.

Cette solution, il faut le dire, convenait à l'insécurité et au conservatisme muré de bien des individus dans la profession. Ceux que Wasserman surnomme les "héros modernes de la discipline" promettaient ainsi d'annihiler les déficiences des systèmes en place sans que le bibliothécaire n'ait, lui, à changer, à évoluer dans sa conception acquise de la bibliothèque, du type d'information disponible et des exigences de son nouveau public. ${ }^{26}$

Le plus curieux de toute cette expérience d'automatisation au sein de la bibliothèque, c'est que le pourquoi même de toute cette opération qui consistait à faire face au défi de l'information éphémère a souvent été fort négligé au profit d'objectifs qui servaient d'abord et avant tout la bibliothèque. Ses grandes victoires au sein de la bibliothèque, l'ordinateur les doit, jusqu'à maintenant, presque exclusivement à des opérations de contrôle (prêt, périodiques, acquisitions, etc.) alors que les activités axées sur le traitement en sont encore à l'étape des balbutiements (ex: télécatalogage). ${ }^{27}$

En résumé, après plus de 15 ans d'intégration de l'ordinateur au sein de la bibliothèque, l'information parvient à l'usager dans des délais qui demeurent sensiblement les mêmes, et avec une qualité d'indexation toujours aussi déficiente. ${ }^{28} \mathrm{De}$ fait, ce qu'on nous propose encore trop souvent, ce sont les mêmes vieilles recettes servies à la moderne. Nous ne sommes plus les seuls à croire qu'il serait

26. Paul Wasserman, The New Librarianship..., p. 12 27. M. E. Maron, "Theoretical librarianship and information science". Information, Part 2, vol. 2, no. 4 (1973), 3

28. Robert S. Taylor, "Innovation in libraries: effect on function and organization", in Conrad H. Rawski, ed. Toward a Theory of Librarianship; Papers in Honor of Jesse Hark Shera, Metuchen, N.J., Scarecrow Press, 1973, p. 455 peut-être temps de s'interroger sur la finalité même des systèmes que nous mettons aujourd'hui en place à si grands frais. ${ }^{29}$

Le but que nous poursuivons n'est pas de jeter l'opprobre universel sur le bibliothécaire contemporain, accroché à un nom qui ne lui sied plus et à des techniques et normes toujours aussi archaïques même si les instruments sont changés. Notre objectif ultime est de souligner, s'il y a lieu, ce que nous croyons être certaines faiblesses et, si possible, tenter de prévoir d'où viendront les prochains développements de sorte que le bibliothécaire ne soit pas, cette fois encore, pris au dépourvu.

Le bibliothécaire n'est pas le seul professionnel à avoir fait le long cheminement historique de sa profession en commettant certaines erreurs d'aiguillage. Le cas des médecins en est un autre exemple: après avoir longtemps combattu ceux que l'on appelle aujourd'hui les chiropraticiens (autrefois appelés "charlatans"), ils ont néanmoins vu ces derniers s'imposer parce qu'ils comblaient, par des techniques nouvelles, un besoin qu'eux n'avaient su prévoir ou assumer.

Ce qui distingue par contre le médecin du bibliothécaire, c'est que, lui, il a appris la leçon. Le nombre d'équipes médicales faisant la navette entre l'Occident et la Chine populaire permet de penser que les acupuncteurs, par exemple, n'auront pas à subir les mêmes tracasseries que leurs confrères "chiros". En un mot, les médecins ont décidé de se réajuster en fonction des besoins et techniques de l'époque, d'apprendre s'il le fallait, mais surtout de faire en sorte que, ce par quoi ils se définissent, spécialistes de la santé, continue d'être de leur ressort et, disons-le, chapeauté par eux.

En continuant de nous identifier à un nom qui ne nous sied plus, à des techniques qui ne rencontrent pas nécessairement les besoins de l'époque, nous prolongeons la confusion sur le service à rendre et nous nous plaçons ainsi, malheureusement, dans une situation qui accentue notre immobilité, diminue notre capacité de prévision et de changement et fera en sorte qu'éventuellement nous risquons de ne constituer qu'un groupe marginal au sein de la famille plus large des spécialistes de l'information. ${ }^{30}$

29. Douglass John Foskett, "The contribution of classification to a theory of librarianship", in Conrad $\mathrm{H}$ Rawski, ed., Toward a Theory..., p. 169

30. Mary Lee Bundy and Paul Wasserman, "Professionalism...", 25 


\section{Perspectives d'avenir}

Si la profession a été soumise jusqu'à aujourd'hui à des transformations extérieures qui l'ont ébranlée jusque dans ses racines, rien ne permet de supposer que la situation est maintenant stationnaire, qu'avec l'avènement de l'automation tous les problèmes sont réglés et, qu'enfin, la bibliothèque a pris le pas. Rien ne permet d'assurer que le moteur de changement à l'origine de tout ceci a atteint aujourd'hui son stade final, que le bibliothécaire peut maintenant espérer respirer quelque temps. Bien au contraire, on peut logiquement penser que le processus va se continuer, que la roue tournera toujours.

Ce qui est dommage dans le cas du bibliothécaire, c'est que, trop souvent, il apparaît comme éternellement bousculé par le changement plutôt que d'en être le moteur conscient. Toujours un pas en arrière, il bouche les trous pendant que d'autres autour de lui créent les "problèmes" de demain, échafaudent les solutions possibles et, éventuellement, combleront le besoin.

Ainsi, pendant que la profession tentait tant bien que mal de récupérer en son sein le non-imprimé, d'autres remplissaient à leur façon, par des méthodes nouvelles, le besoin existant d'une information à caractère éphémère qui soit traitée rapidement. Et pendant qu'aujourd'hui nous tentons, tant bien que mal encore, de rattraper le temps perdu en essayant de présenter un produit qui satisfasse aux normes minimales de l'information éphémère en intégrant l'ordinateur au processus, pendant ce temps, il y a beaucoup à parier que nous manquons encore une fois le bateau, que les véritables questions et besoins qui tourmentent aujourd'hui l'opinion et où nous sommes absents seront encore là nos problèmes de demain.

Nous n'avons pas ici l'intention de jouer au prophète ou au spécialiste en prospective. Néanmoins, nous aimerions tracer rapidement les grandes lignes de ce que peuvent être les défis de notre décennie pour notre profession.

Étant acquis (pour nous, tout au moins) que l'objet de la profession est l'information enregistrée et que le bibliothécaire est un spécialiste de cette information; étant acquis que l'information vieillit très vite et que le bibliothécaire se doit de rencontrer certaines normes minimales en terme de disponibilité de l'information tout en y assurant un accès beaucoup plus sophistiqué, quelle est la prochaine étape?
$\mathrm{Si}$, par analogie, l'on examine l'évolution récente de la plupart des groupes professionnels qui nous entourent, on ne peut manquer d'être frappé par cet effort de redéfinition sociale, imposé ou voulu, auquel ils sont soumis. Dans les secteurs typiquement scientifiques, on assiste à une redéfinition du terme "progrès", progrès qui, depuis la révolution industrielle, primait sur tout. Désormais le progrès, peut-être pour la première fois dans I'histoire, doit composer avec les besoins perçus des citoyens. On n'échafaude plus ce qu'on veut, où et quand on veut. La société se donne des normes qui la protègent. De plus en plus on tente d'asservir le progrès, de faire en sorte qu'il serve d'abord et avant tout aux besoins formulés de l'individu.

Le "progrès" devient alors conditionné à un ensemble de critères sociaux jusqu'alors complètement négligés. L'architecte qui conçoit un édifice en hauteur se préoccupe, souvent parce qu'on l'y oblige, de l'impact social de sa construction. Le chimiste se sentira obligé de justifier socialement sa recherche, ne serait-ce que pour obtenir de nouvelles subventions. De nouvelles professions surgissent, alimentées par cet impact des préoccupations sociales sur le monde scientifique. Pensons aux écologistes, environnementalistes, etc. Le progrès scientifique, longtemps perçu comme une fin en soi, devient de plus en plus subordonné à des critères extérieurs à la stricte rationalité scientifique.

La population s'est sentie détentrice de certains pouvoirs, pouvoirs qu'elle monnaie littéralement en retour d'avantages qu'elle perçoit comme nécessaires, voire indispensables, avantages qu'elle formule en terme de droits: droit à la santé, à la justice, au travail, etc.

Le cas des médecins et des avocats est, à cet égard, particulièrement significatif. Ces deux professions, traditionnelles s'il en est, se sont vues soumises au cours des dernières années à des transformations radicales, le plus souvent d'ailleurs contre leur gré. Au médecin qui, traditionnellement, se percevait comme un "guérisseur de maladie", on impose plus: le droit à la santé. Chaque individu, qu'il soit riche ou pauvre, se proclame éligible à la santé. La médecine préventive, entre autres, est une illustration de ce bouleversement dans l'approche élaborée.

Le juriste fut placé dans le même dilemme. Le droit à la justice que depuis peu s'octroient les gens a vraiment secoué et risque de secouer encore davantage la profession. Pen- 
sons, entre autres, à l'instauration des cliniques juridiques.

Identifier des besoins et exiger qu'ils soient comblés impliquent nécessairement, comme corollaire, de la part du gouvernement et des groupes qui détiennent le pouvoir l'élaboration de politiques et de services aptes à satisfaire ces besoins. Ainsi, le gouvernement a lui-même établi des normes et édifié des services de plus en plus nombreux et coûteux. La population exigeant encore davantage, il a dû, et souvent avec le plus grand déplaisir, agir auprès des dispensateurs de ces services jugés indispensables par la population.

Il va donc de soi que, soumises au feu nourri à la fois de la population et des gouvernements, plusieurs professions ont dû et devront sans doute encore réévaluer leur activité, l'envisageant essentiellement dans une optique de service à la population.

Qui plus est, c'est dans la mesure où un groupe donné peut justifier à la population, et ainsi au gouvernement, un rôle de dispensateur d'un service qu'elle perçoit comme important, qu'il atteint, moyennant certains critères minima comme l'éducation, au statut suprême de "profession". En un mot, l'accès au professionnalisme, ou tout simplement l'amélioration du statut professionnel, passe et risque de passer de plus en plus par la place publique.

Les exemples de cet état de fait sont quotidiens. Que ce soient les pharmaciens qui tiennent à récupérer la vente des "Rolaids" au nom de l'intérêt public; les avocats qui défendent le droit de l'individu à leurs services face à l'arbitraire possible (ex: les petites créances); les professeurs qui, au nom du bien de l'enfant, veulent voir réduire le nombre d'étudiants admis à chacun de leurs cours, tous sentent la nécessité de traduire en terme de besoin, de droit et de service à la population des améliorations potentielles à leurs conditions de travail, leur statut ou même leurs privilèges.

Comment le bibliothécaire qui, lui aussi, se veut professionnel manœuvre-t-il à travers ces demandes et ces exigences? Fort mal, encore une fois. Pendant que l'on discute automation, que l'on tente de régler tant bien que mal aujourd'hui les problèmes qui appartiennent déjà à une autre époque, pendant ce temps la population traduit son besoin d'informations en téléphonant à madame Unetelle, en écrivant à monsieur Untel, ou tout simplement en exigeant que se créent autour d'elle les organismes aptes à la renseigner. Information-Canada et Communication-Québec sont de ceux-là, sans parler des nombreux centres d'informations sur ceci ou cela qui sont créés un peu partout. Et où est le bibliothécaire pendant ce temps, ce spécialiste de l'information enregistrée?

Encore une fois, le même scénario se répète. Quand le bibliothécaire aurait dû répondre aux besoins du milieu pour faire face au défi du non-imprimé, il n'a pas bougé et d'autres s'en sont chargés; quand il aurait dû déceler les besoins du milieu pour faire face au défi de l'information éphémère, il tentait tant bien que mal d'intégrer le non-imprimé à sa bibliothèque et d'autres, encore là, ont répondu à ces besoins. Aujourd'hui, quand il devrait trouver les moyens de faire face au défi d'une information accessible à tous, d'une profession axée sur le service à la population, il tente avec plus ou moins de succès de régler le problème de l'information éphémère et d'autres, encore là, comblent le besoin d'informations qui se manifeste au sein d'une population qui la réclame parce que bouleversée, charroyée par une société de plus en plus complexe, stressante et compétitive.

Les complexes qui assaillent les bibliothécaires face à une telle situation sont remarquables. Dans la profession, on a peur du monde, peur d'offrir un service qui pourrait être apprécié et, par là, entraîner des pressions sur l'organisation et déranger les vieilles habitudes, ce que Wasserman identifie à la psychologie de la "petite entreprise" qui demeure petite parce que sa situation lui offre une garantie contre le changement. ${ }^{31}$ On a peur de devoir être identifié à ce que l'on n'est pas, par exemple au travailleur social si l'on indique à quelqu'un où se trouve l'hôpital le plus proche, etc. Le mot d'ordre dans la profession est de faire en sorte qu'on vous oublie, qu'on ne parle pas de vous.

Les conséquences d'une pareille attitude sont énormes, on le conçoit aisément. C'est d'abord, encore une fois, rater le rendez-vous avec les besoins de son époque, c'est accumuler un retard qu'il sera plus tard très difficile de rattraper, à moins qu'on décide que ce n'est pas de son ressort, que le bibliothécaire n'a rien à faire là-dedans, etc.

Si tel était le cas, il faut bien comprendre que ce serait tuer à jamais toute possibilité de développement de la bibliothèque dite publique au Québec, car la population et le gouvernement n'accepteront plus de financer

31. Paul Wasserman, The New Librarianship..., p. 34. 
des services ouverts aux besoins particuliers de l'élite éprise de lecture. ${ }^{32}$

Rater ce rendez-vous, c'est aussi reléguer aux oubliettes, pour longtemps encore, toute aspiration à quelque statut professionnel que ce soit pour les gens œuvrant dans cette profession.

Le bibliothécaire est à une croisée des chemins: ou il accepte de se redéfinir socialement, de s'identifier aux besoins d'informations de la population et de rendre effectivement le service en question, ou il recule encore une fois d'un cran, replié sur lui-même et sa bibliothèque moribonde.

Les implications du premier choix sont de nature à bouleverser beaucoup d'attitudes acquises au sein de la profession. S'identifier aux besoins d'informations des gens, c'est promouvoir le droit à l'information pour la population; c'est rejoindre le journaliste, le recherchiste et les autres groupes œuvrant au sein de la grande famille de l'information et participer aux mêmes combats pour une accessibilité totale de tous à l'information. C'est évidemment s'identifier comme spécialiste de l'information dans le secteur de notre compétence, l'information enregistrée, et faire le "marketing" en conséquence auprès du public appelé à bénéficier de nos services.

Au sein du groupe plus restreint des spécialistes de l'information en registrée, c'est briser les barrières existantes entre les spécialités en s'identifiant, tous et chacun (bibliothécaires, documentalistes, informatistes, etc.) à une profession unique qui intègre et projette publiquement ce qui constitue, de fait, l'objet de notre profession et n'entraîne pas de confusion au sein de la population à desservir. ${ }^{33}$

Au sein du monde des bibliothèques, c'est abolir les barrières encore existantes déterminant l'accès à divers types de bibliothèques, centres de documentation ou autres. Toutes les bibliothèques ne peuvent mettre en place des collections aptes à satisfaire les besoins de toutes les clientèles. Cependant, tous les bibliothécaires ou ce qu'ils pourraient devenir, devraient être en mesure de diriger l'usager vers la personne ou les centres spécialisés aptes à rendre le service en question, tout comme on attend du médecin qu'il puisse

32. Ibid., p. 13

33. La création de l'ASTED est, à cet égard, tout à fait remarquable. C'est un premier pas dans la voie d'une intégration de la profession autour du contenu et non du contenant. orienter vers les spécialistes s'il ne peut luimême rendre le service demandé.

Au sein de la bibliothèque, c'est créer des structures qui favorisent, chez les bibliothécaires, des comportements de professionnels, c'est-à-dire de gens ayant à cœur le service à leur clientèle plutôt que l'organisation bureaucratique. À cet égard, la traditionnelle structure par services (acquisitions, référence, catalogage, sélection) aurait avantage à être repensée. On pourrait lui adjoindre, tel que le propose Kaser, ${ }^{34}$ une structure parallèle, celle-là plus professionnelle, constituée, par exemple, d'unités naturelles opérant tout au long de la chaîne de traitement où les tâches seraient plus polyvalentes, les interactions plus nombreuses entre les personnes œuvrant dans une même spécialité. Ainsi, une chaîne en sciences sociales pourrait regrouper la ou les personnes chargées de la sélection dans le secteur, des acquisitions, du catalogage et de la référence; tous ces gens constitueraient une équipe capable de régler elle-même les problèmes de son secteur, en fonction des besoins de sa clientèle, le but essentiel étant de créer, au sein de l'équipe, un climat de service à la clientèle où tous se sentiraient impliqués. Le rôle de l'actuel directeur de service deviendrait celui d'un animateur, d'un stimulant, d'un générateur d'idées dans la spécialité qui est la sienne et, bien sûr, un élément de contrôle dans l'application de certaines normes et comportements minimaux indispensables à la bonne marche de l'organisation.

Il est complètement illusoire de rêver à un statut professionnel quelconque si les individus de cette profession n'envisagent pas un jour ou l'autre de se comporter comme tel, et ceci implique d'abord de répondre aux besoins des gens, de se définir en terme de service disponible à la population et de créer les structures qui favorisent un tel climat pour tous et chacun dans la profession.

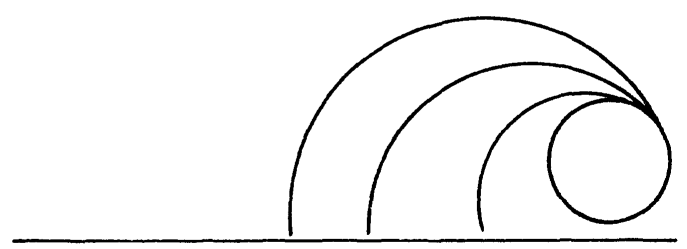

34. David Kaser, "Modernizing the university library structure», College and Research Libraries, vol. 31, no. 4 (July 1970), 228-229. 